\title{
Treatment with Immunosuppressants FTY720 and Tacrolimus Promotes Functional Recovery after Spinal Cord Injury in Rats
}

\author{
Jie Zhang, ${ }^{1 *}$ Ailiang Zhang, ${ }^{1 *}$ Yu Sun, ${ }^{1}$ Xiaojian Cao ${ }^{1}$ and Ning Zhang ${ }^{1}$ \\ ${ }^{1}$ Department of Orthopedics, First Affiliated Hospital, Nanjing Medical University, Nanjing, Jiangsu Province, \\ China
}

\begin{abstract}
Spinal cord injury (SCl) occurs frequently and is a leading cause of permanent disability in young adults. Many immune inhibitors including tacrolimus (FK506) are shown to be helpful in the regeneration of neural tissue following spinal cord injury. FTY720 belongs to a new class of immunosuppressants. The combination of FTY720 and tacrolimus has been reported to elicit synergistic immunosuppresive effects in rat allograft models without causing critical adverse effects. This study was to determine whether the combination of FTY720 and tacrolimus is superior to FTY720 or tacrolimus alone in the treatment of SCI. Forty-eight rats were subjected to a weight-drop contusion at the tenth thoracic level (a 10-g rod dropped from a height of $25 \mathrm{~mm}$ ). At $30 \mathrm{~min}$ after the operation, they were randomly divided into four groups and received treatment with either FTY720 $(0.5 \mathrm{mg} / \mathrm{kg})$, tacrolimus $(0.5 \mathrm{mg} / \mathrm{kg}), \mathrm{FTY} 720$ + tacrolimus $(0.5 \mathrm{mg} / \mathrm{kg}$ and $0.5 \mathrm{mg} / \mathrm{kg}$ respectively) or saline via gavage. Functional recovery was evaluated during 42 days after $\mathrm{SCl}$ via open-field test, inclined plane test, footprint analysis, somatosensory evoked potentials (SSEPs), and electron microscopic analysis. Rats from three treatment groups showed significantly better locomotor functional outcomes, higher SSEP amplitude, shorter SSEP latency, and milder pathological changes compared with those of control group. Moreover, rats treated with a combination of FTY720 and tacrolimus demonstrated significantly greater functional recovery by day 14 after $\mathrm{SCl}$ than those treated with either FTY720 or tacrolimus alone. These results suggest that the combination of FTY720 and tacrolimus could be a potentially effective therapeutic strategy to treat SCI. — spinal cord injury; FTY720; tacrolimus; immune inhibitor; lymphocyte.

Tohoku J. Exp. Med., 2009, 219 (4), 295-302. (C) 2009 Tohoku University Medical Press
\end{abstract}

Spinal cord injury (SCI) is a devastating condition affecting primarily young males. The annual incidence of SCI in the United States is 12,000/year, with a prevalence of over 250,000 persons. Nearly $80 \%$ of individuals sustaining SCI are male (Dorsher and McIntosh 2009). Treatment for individuals with SCI in the USA estimatedly costs more than $\$ 7$ billion per year. In China, the incidence of SCI has been increased markedly in the past 10 years. Most SCIs are incomplete. In some patients whose motor and sensory paralysis even appears complete, spared neural tissue has also been found post-mortem (Saadoun et al. 2008).

Recent experimental studies in laboratory animals have shown that secondary injury of SCI can be decreased by exogenous interventions with drugs to diminish repulsive barriers (Rossignol et al. 2007). It occurs in a delayed yet progressive fashion and involves cellular and biochemical events that initiate cascades culminating in tissue damage and cell death (Dumont et al. 2001). During the process, the immune response plays an important role in the mechanism of spinal cord functional regeneration after its injury.
Hauben et al. (2003) suggested that stimulating the immune response is helpful in functional recovery of SCIs in some circumstances. On the other hand, in vivo and in vitro studies have shown that many immune inhibitors, such as rapamycin toxin, cyclosporine $\mathrm{A}$, and tacrolimus are helpful in the regeneration of central and peripheral axons (Shiraishi et al. 2001; Gillon et al. 2003). Tacrolimus can promote early physiological function recovery in transplanted allogeneic limbs (Dubernard et al. 2003) and improve the limbs' locomotor function after acute SCI (Madsen et al. 1998; Voda et al. 2005; Pan et al. 2006a). Tacrolimus has been considered as a potential therapy for SCI, since it has greater neuroprotective effects than that of methylprednisolone, a common drug used in the treatment of SCI (Kaymaz et al. 2005; Lopez-Vales et al. 2005). However, immunotherapeutic application of tacrolimus has shown many adverse side effects including nephrotoxicity, diabetogenicity, and the development of lymphoproliferative disorders (Gruessner 1997), which limit the wide use of tacrolimus.

Since 1994, a new type of immune inhibitor called

Received August 18, 2009; revision accepted for publication October 22, 2009. doi:10.1620/tjem.219.295

*Jie Zhang and Ailiang Zhang contributed to this work equally.

Correspondence: Xiaojian Cao, Department of Orthopedics, First Affiliated Hospital, Nanjing Medical University, Guangzhou Road

300, Nanjing, 210029, Jiangsu Province, China.

e-mail: xiaojiancao@gmail.com 
FTY720 has been found (Fujita et al. 1994). Compared with the traditional immune inhibitors, FTY720 has the advantages of stronger immune inhibiting effects, good synergistic effects with other immunosuppressive drugs, little side effects, broad treatment scope and so on. The signature feature of FTY720 is its ability to rapidly reduce blood lymphocytes, especially $\mathrm{T}$ cells, as a consequence of $\mathrm{S}_{1} \mathrm{P}_{1^{-}}$ mediated retention in the peripheral lymph nodes (Lo et al. 2005). It is notable that FTY720 not only spares $\mathrm{CD} 4^{+} \mathrm{CD} 25^{+} \mathrm{T}$-regulatory cells but also restores their functional activity (Daniel et al. 2007). It has been hypothesized that the apoptotic cell death of lymphocytes and the acceleration of lymphocyte homing decrease the number of lymphocytes (Chiba et al. 1998; Yanagawa et al. 1998). Emerging evidence suggests that FTY720 could be effective in treating central nervous system (CNS) injury by influencing the blood-brain barrier and glial repair mechanisms that could ultimately contribute to the restoration of neural function (Balatoni et al. 2007; Foster et al. 2007). Moreover, the unique mechanism of FTY720 allows for its use in combination with other immunosuppressants (Tamura et al. 2000). In fact, the combination of FTY720 and tacrolimus produced additional immunosuppressive effects in rat allograft models (Hoshino et al. 1999; Tamura et al. 2000) and resulted in fewer changes in kidney structure and lower blood glucose levels than did tacrolimus alone (Gallo et al. 2006; Lopes et al. 2008). The present study investigated whether the combination of FTY720 and tacrolimus could result in a synergistic and beneficial effect on functional recovery after SCI.

\section{Materials and Methods}

\section{Animals}

The experiment was performed in accordance with the 'Guide for the Care and Use of Laboratory Animals' published by the Chinese National Institutes of Health. Protocols were approved by the Animal Ethics Committee of Nanjing Medical University. Since nearly $80 \%$ of individuals sustaining SCI are male, only male rats were used in the present study. Forty-eight pre numbered Sprague-Dawley (SD) male rats weighing an average of between 280 and $320 \mathrm{~g}$ were randomly divided into four groups via simple random sampling without repeats approach. The four groups are as following: an FTY720-treated group, a tacrolimus-treated group, an FTY720 + tacrolimus-treated group, and a saline-treated control group. All rats were housed in the same environment with free access to standard rodent chow and water, at a constant temperature of $23-28^{\circ} \mathrm{C}$ and a relative humidity of $60-75 \%$.

\section{Reagents}

FTY720 was purchased from Yoshitomi Pharmaceutical lndustries Ltd. (Tokyo, Japan); tacrolimus was purchased from Beijing Novartis Pharma Ltd. (Beijing, China).

\section{Surgical procedure}

Spinal cord injury was induced using a weight-drop device developed at New York University (Gruner 1992) following a protocol developed by a multicenter consortium (Multicenter Animal Spinal
Cord Injury Study; Basso et al. 1995), which has been reported previously (Hauben et al. 2001). Rats were anesthetized with pentobarbital $(50 \mathrm{mg} / \mathrm{kg}$, i.p.), and a laminectomy was performed at the T9-T10 level. After the spinous processes of T8 and T11 were clamped to stabilize the spine, the exposed dorsal surface of the cord was subjected to a weight-drop impact using a 10-g rod dropped from a height of 25 $\mathrm{mm}$. The muscles and skin were then closed in layers. After the injury, the rats were placed in a temperature- and humidity-controlled chamber. Manual bladder expression was performed at least three times daily until reflex bladder emptying was established.

\section{Reagent Administration}

The optimal time to administer the reagent was referred to Lopes-Vales et al's studies (Lopez-Vales et al. 2005) and the chosen dose was based on previous studies with minor modifications (Cao et al. 2005; Pan et al. 2006b, papers in Chinese). In above studies, both time course and dose curve were conducted and results indicate 30 min post-surgery and $0.5 \mathrm{mg} / \mathrm{kg}$ of drug concentration are the optimal conditions to exam the efficacy of the drugs in rat SCI models. Thirty minutes post-operation, rats in three treatment groups were administered, by gavage, either FTY720 (0.5 mg/kg, in PBS, Saline), tacrolimus $(0.5 \mathrm{mg} / \mathrm{kg}$ in PBS, Saline) or FTY720 + tacrolimus $(0.5 \mathrm{mg} / \mathrm{kg}$ and $0.5 \mathrm{mg} / \mathrm{kg}$ respectively). Rats in the control group were administered saline at same dose $(0.5 \mathrm{mg} / \mathrm{kg})$ by gavage.

\section{Functional Recovery Evaluation}

Following three types of functional tests were used to assess the functional recovery. Each test was observed by two independent investigators.

Open-field test. The open-field locomotion test assesses movement, weight support and coordination. It was scored using the standardized Basso, Beattie and Bresnahan (BBB) locomotor scoring system (Basso et al. 1995). BBB scores range from 0 (flaccid paralysis) to 21 (normal gait). Rats were acclimated to the testing environment ( $90 \mathrm{~cm}$ diameter plastic wading pool; $4 \mathrm{~cm}$ height) prior to testing. The test was performed before operation and at days 3, 7, 14, 21, 28, 35 and 42 post-operation. BBB scores were averaged for each group.

Inclined plane test. This test evaluates the maximum angle on the inclined plane upon which each animal can maintain a stable position for 5 seconds (Rivlin and Tator 1977). Rats were placed on a board that was incrementally raised to increase angles. The test was performed before operation and at days 3, 7, 14, 21, 28, 35 and 42 post-operation. Angle scores were averaged for each group.

Footprint analysis. The walking patterns of the hindlimbs were recorded with ink during continuous locomotion across a $90 \mathrm{~cm}$ runway. The stride length on each side and the stride width between the two sides were calculated (Metz et al. 2000; Li et al. 2004; Fu et al. 2007). This test was performed at 42 days post-operation. Stride length and stride width were averaged for each group.

\section{Electrophysiological assessments}

Electrophysiological tests were carried out before operation and at days 21 and 42 post-operation. Somatosensory evoked potentials (SSEPs) were conducted through the spinal cord to the sensory cortex of the brain and recorded (Jou et al. 2000). The rats were anesthetized with sodium pentobarbital $(25 \mathrm{mg} / \mathrm{kg}$, i.p.), and placed in a prone position over a warmed flat coil to maintain skin temperature above $34^{\circ} \mathrm{C}$. Subdermal electrodes stimulated nerve impulses from the tibial nerves of both hindlimbs (stimuli trains in sets of 200 at $3 \mathrm{~Hz}$; stimu- 
lus amplitude $3 \mathrm{~mA}$ square wave, $200 \mu \mathrm{s}$ in duration). The active electrode for the SSEP recording was placed into the interspinous ligament at T10. The reference electrode was placed subcutaneously at approximately the same level. The signals were amplified, filtered and displayed on the oscilloscope (Keypoint EMG and EP systems, Medtronic, Skovlunde, Denmark) to measure both the amplitude and the latency of the evoked potentials. Amplitude and latency of SSEPs were averaged for each group.

\section{Electron microscopy (EM)}

At 21 days post-operation, five rats randomly selected via simple random sampling method on pre-labeled rats in each group were euthanized and perfused intracardially with saline, followed by $2 \%$ glutaraldehyde and $4 \%$ paraformaldehyde in $0.1 \mathrm{M}$ sodium cacodylate buffer, $\mathrm{pH}$ 7.4. Immediately after perfusion, their spinal cords were removed and postfixed in the same fixative overnight at $4^{\circ} \mathrm{C}$. The spinal cord segment at the injury epicenter was sliced into $1 \mathrm{~mm}$ pieces, postfixed for $2 \mathrm{~h}$ in $1 \% \mathrm{OsO}_{4}$ in $0.1 \mathrm{M}$ cacodylate buffer, dehydrated in graded ethanol solutions and embedded in Epon-812 (Ernest Fullam, Latham, NY). One micrometer semi thin plastic sections were cut and stained with $1 \%$ toluidine blue before examination with a Nikon Eclipse TE300 microscope equipped with a Spot RT Color CCD camera. For EM, blocks were trimmed and sections were cut at $100 \mathrm{~nm}$, mounted on copper grids, stained with uranyl acetate and lead citrate and viewed with a JEOL (Tokyo, Japan) Jem 1200 EX transmission electron microscope.

\section{Statistical analysis}

All functional and electrophysiological measurements were carried out in a double blinded manner using codes for both the animals and the histologic slides. The statistical analysis was performed using SPSS software 13.0. Data were expressed as mean \pm standard error of the mean (SEM). Statistical comparisons among the four groups were made using analysis of variance (ANOVA) with the post hoc Scheffé test for multiple comparisons. A probability level of less than 0.05 was considered statistically significant.

\section{Functional Recovery}

Before operation, all groups received mean BBB scores of 21, indicating normally ambulating rodents. All groups received mean BBB scores of 21 at 3 days postoperation. Both the FTY720 + tacrolimus-treated group (at 7 days post-operation) and the FTY720- and tacrolimustreated groups (at 14 days post-operation) showed significantly improved hindlimb performance compared to the saline-treated control group $(P<0.05)$. This superior recovery continued throughout the survival period. The FTY720 + tacrolimus-treated group demonstrated a higher mean BBB score $(P<0.05)$ than those of the FTY720- and tacrolimus-treated groups at 14 days post-operation. There was no statistical difference in mean BBB scores between the FTY720- and the tacrolimus-treated groups (Fig. 1).

Inclined plane testing was conducted to assess the functional integrity of subsets of supraspinal pathways. Preoperatively, all groups showed mean angle scores of approximately 65 degrees. Post-operation, the mean angle scores decreased, and then gradually increased for all

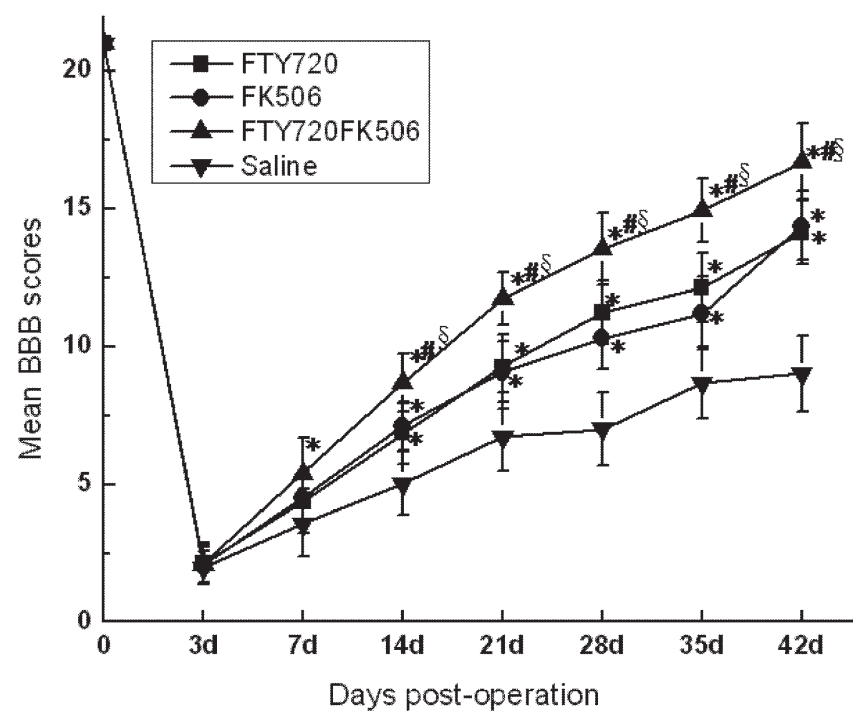

Fig. 1. BBB scores by Open-field test. Plots showing progression of the open-field locomotor activity evaluated by BBB score over 42 days post-operation. $* P<0.05$ versus the saline-treated control group; ${ }^{\#} P<0.05$ versus the FTY720-treated group; ${ }^{\S} P<0.05$ versus the tacrolimustreated group. Error bars represent SEM.

groups. There was no difference in mean angle scores among the four groups at 3 days post-operation (Fig. 2). All three treatment groups demonstrated significantly higher mean angle scores than that of saline-treated control group $(P<0.05)$ at 7 days post-operation (Fig. 2$)$. Moreover, the FTY720 + tacrolimus-treated group showed a higher mean angle score than those of the FTY720- and tacrolimus-treated groups $(P<0.05)$ at $14-42$ days post-operation (Fig. 2$)$. No differences in mean angle scores were observed between the FTY720- and the tacrolimus-treated groups ( $P$ $>0.05$ ) post-operation (Fig. 2).

To further characterize the locomotion recovery, footprint analysis were performed for the four groups. Analysis of hindpaw footprints demonstrated a greater mean stride length and a shorter mean stride width in FTY720 + tacrolimus-treated group than in the other three groups at 42 days post-operation $(P<0.05)$ (Fig. 3). No differences were found in the mean stride width among the FTY720-, tacrolimus- and saline-treated control groups $(P>0.05)$, although there were significantly greater mean stride lengths in the FTY720- and the tacrolimus-treated groups than in the saline-treated control group $(P<0.05)$ (Fig. 3).

\section{Electrophysiological results}

Electrophysiological recovery was evaluated by means of the amplitude and latency of SSEPs. The amplitude of the SSEPs indicates whether the sensory spinal tracts are disrupted. The normal value of SSEPs amplitude is about 28-32 mV. The latency of the SSEPs shows the impulse conduction of the spared axonal pathways. The normal value of SSEPs latency is about 4 ms. Reduced mean amplitude and lengthened mean latency in SSEPs were observed 


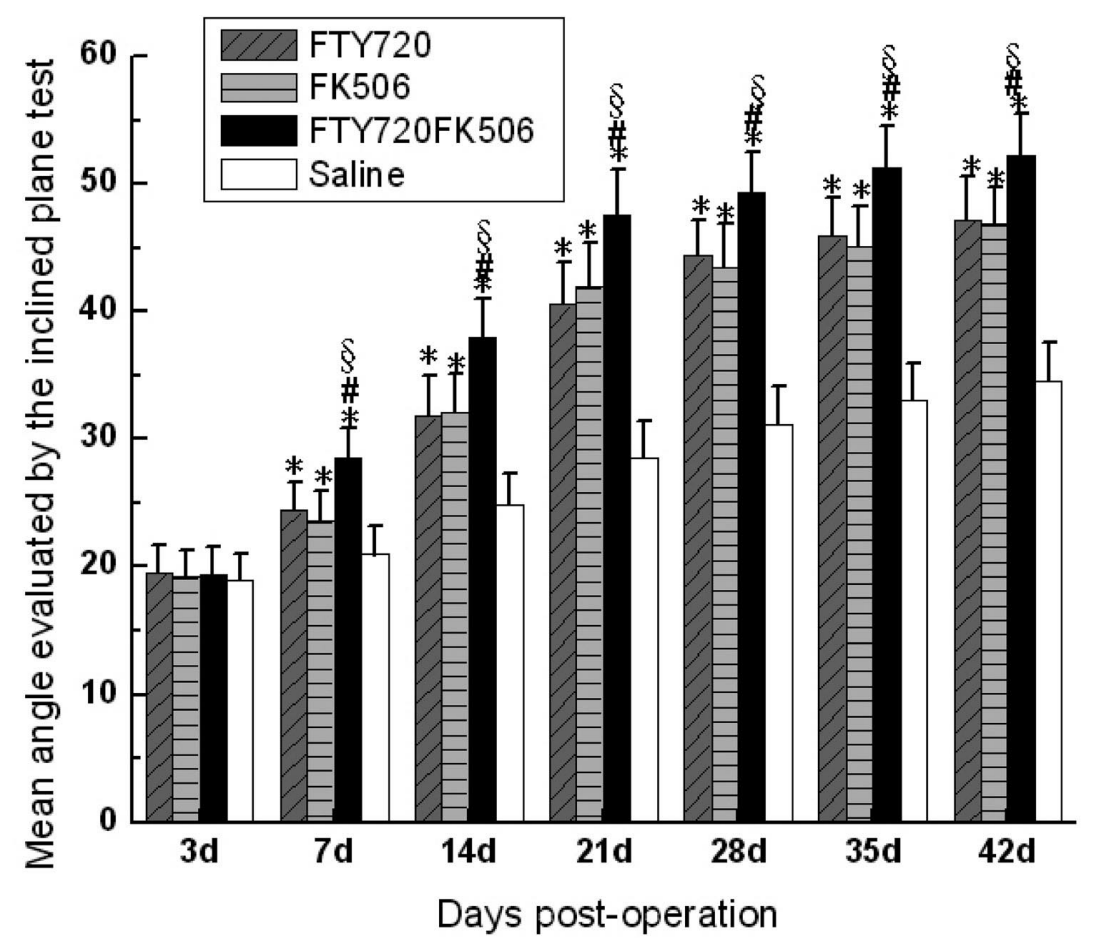

Fig. 2. Angle scores by inclined plane test. Plots showing progression of the maximum angle evaluated by the inclined plane test over the 42 days post-operation. $* P<0.05$ versus the saline-treated control group; ${ }^{\#} P<0.05$ versus the FTY720treated group; ${ }^{\S} P<0.05$ versus the tacrolimus-treated group. Error bars represent SEM.

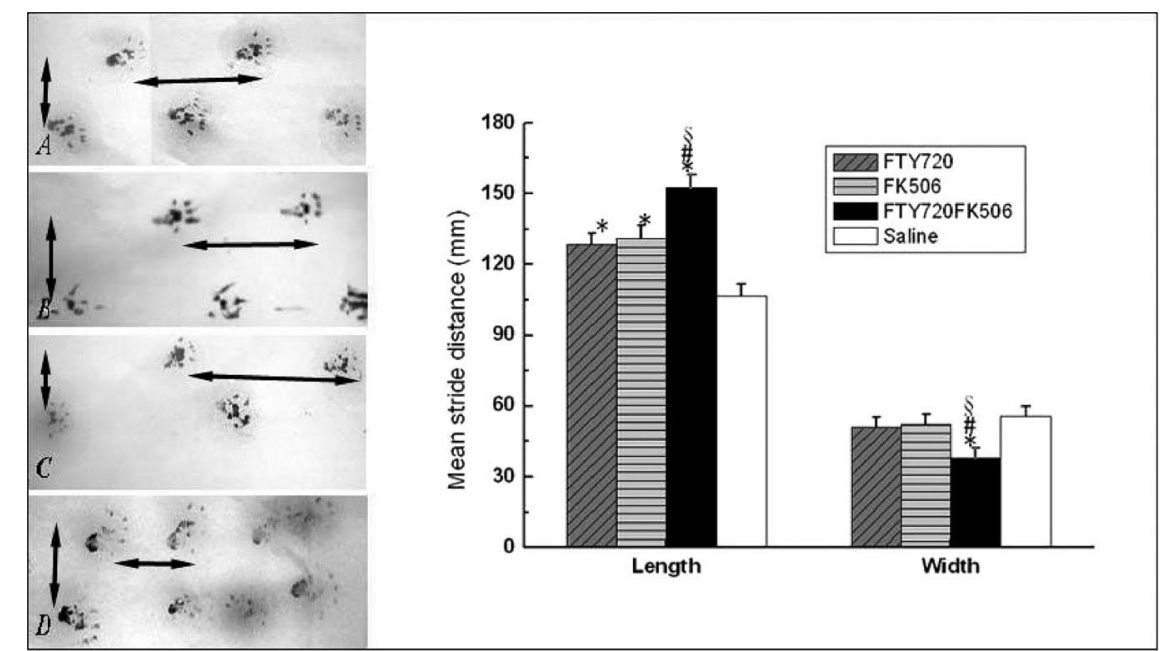

Fig. 3. Footprint analysis. Plots showing the analysis of hindpaw footprints started at 42 days post-operation. A-D: The examples of the representative footprints from rats in the FTY720-treated group (A), in the tacrolimus-treated group (B), in the FTY720 + tacrolimus-treated group (C) and in the saline-treated control group (D). E: Footprint analysis reveals a greater mean stride length and a shorter mean stride width in the FTY720 + tacrolimus-treated group than in the other groups. $* P<0.05$ versus the saline-treated control group; ${ }^{\#} P<0.05$ versus the FTY720-treated group; ${ }^{8} P<0.05$ versus the tacrolimus-treated group. Error bars represent SEM.

in all four groups post-operation, indicating that the motor spinal tracts had been partially disrupted. The FTY720-, tacrolimus- and FTY720 + tacrolimus-treated groups showed significantly higher $(P<0.05)$ mean amplitude and shorter $(P<0.05)$ mean latency of SSEPs compared with those of saline-treated control group at 21 and 48 days post- operation. Moreover, the FTY720 + tacrolimus-treated group showed a significantly higher $(P<0.05)$ mean amplitude and a shorter $(P<0.05)$ mean latency of SSEPs compared with those of the FTY720- and tacrolimus-treated groups. Conversely, there were no significant differences $(P$ $>0.05)$ in either mean amplitude or mean latency of SSEPs 


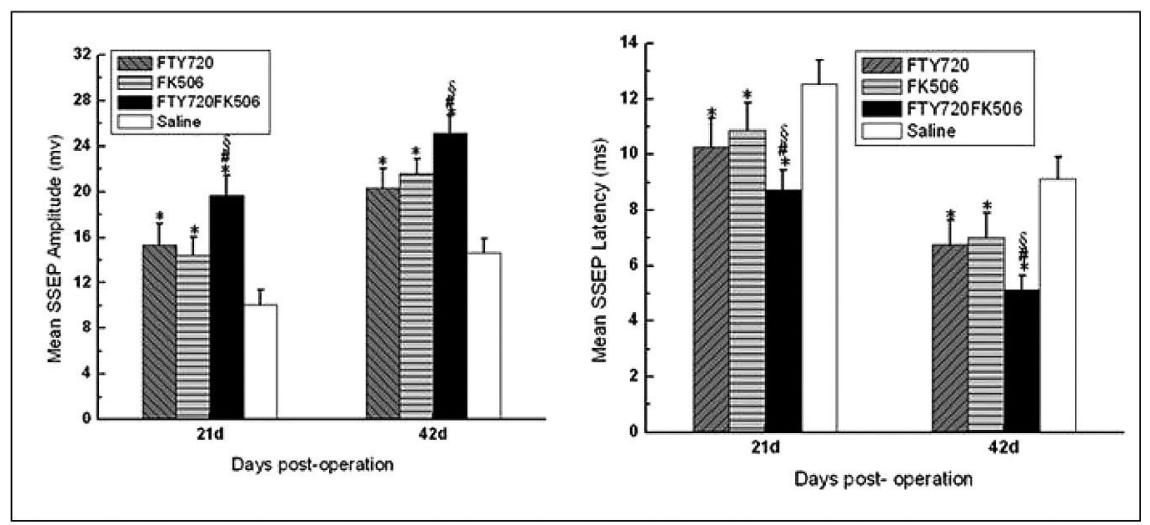

Fig. 4. Outcome by somatosensory evoked potentials. Plots showing the mean amplitude (A) and the mean latency (B) of recovered somatosensory evoked potentials (SSEPs) recorded at 21 and 42 days post-operation. $* P<0.05$ versus the saline-treated control group; ${ }^{\#} P<0.05$ versus the FTY720-treated group; ${ }^{8} P<0.05$ versus the tacrolimus-treated group. Error bars represent SEM.
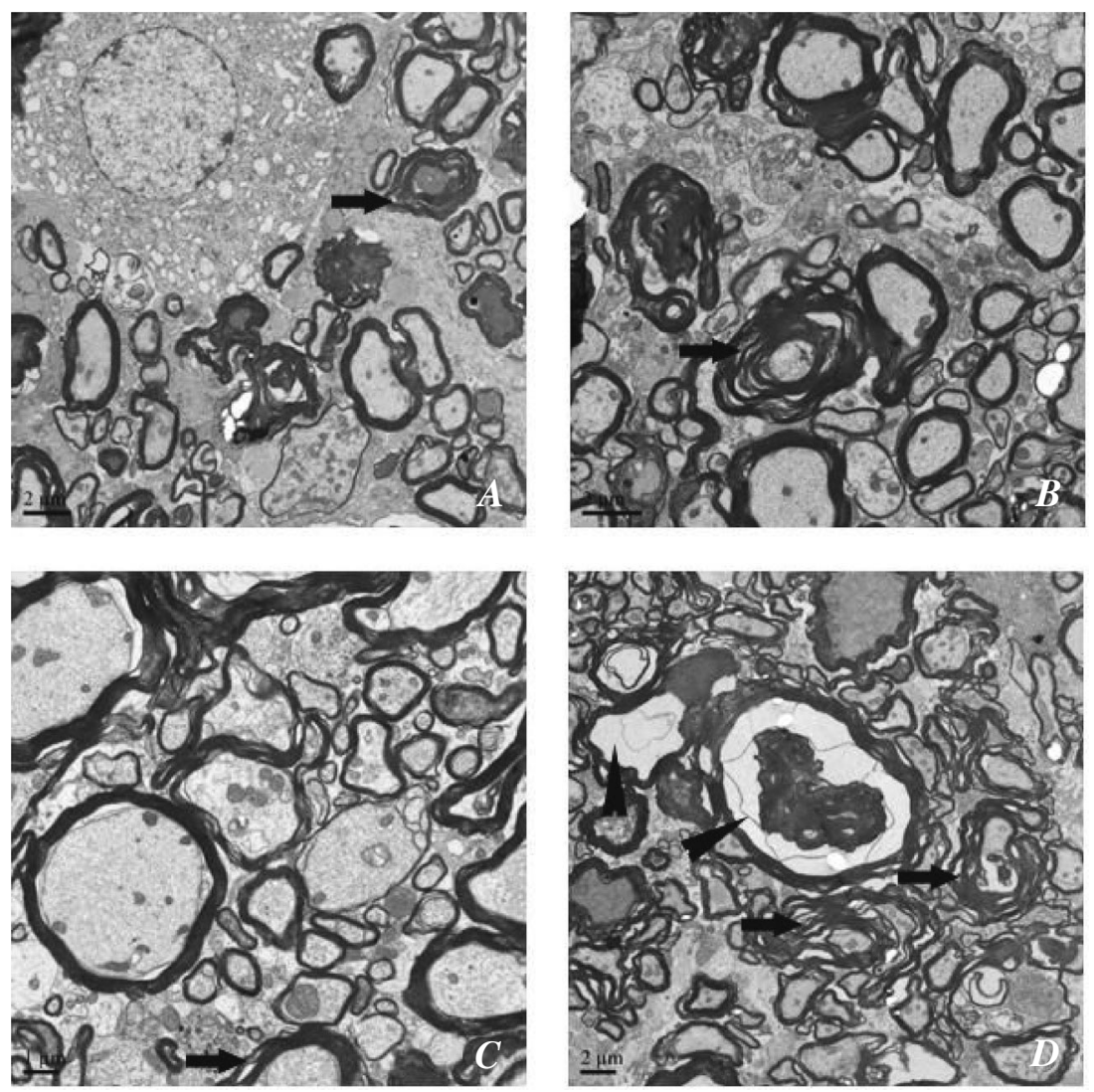

Fig. 5. Representative electron micrographs showing axons and myelin of spinal cord sections taken at 21 days post-operation. EM from the FTY720-treated group (A) and EM from the tacrolimus-treated group (B) show some loss of myelin compaction and intramyelinic vacuolization (arrow). EM from the FTY720 + tacrolimus-treated group (C) shows relatively normal looking axons with slight loss of myelin compaction (arrow). EM from the saline-treated control group (D) shows swollen axons, disruption in myelin structure (arrowheads) and obvious loss of myelin compaction and intramyelinic vacuolization (arrow). Scale bar $=2 \mu \mathrm{m}$ in A, B, D and $1 \mu \mathrm{m}$ in C.

between the FTY720- and tacrolimus-treated groups (Fig. $4 \mathrm{~A}, 4 \mathrm{~B})$.

\section{Histological evaluation}

Electron microscopic studies were performed to deter- mine the effects of FTY720, tacrolimus and FTY720 + tacrolimus on axons and myelin after SCI. At 21 days postoperation, there were only a few pathological changes in the FTY720 + tacrolimus treated group. The axons appeared as slightly oval structures. A darkly stained rim of relatively 
compact myelin, proportional to the axon diameter, surrounded each axon with only a little intramyelinic vacuolization (Fig. 5C). In contrast, there was varying degrees of change in the other three groups after SCI. The most notable change in the appearance of the tissue was the formation of swollen axonal profiles, a decrease in the number of healthy-looking, large-diameter axons, and a disruption in myelin structure. Many axons appeared to have lost their round or oval shapes. This change was most obvious in the saline-treated control group. The white matter showed overt splitting of the myelin lamellae, evident loss of myelin compaction and much intramyelinic vacuolization (Fig. 5D). In the FTY720-treated group, axons retained their round or oval shapes but there was intramyelinic vacuolization and some loss of myelin compaction (Fig. 5A). The changes in the tacrolimus-treated group were similar to those observed in the FTY720-treated group, but the loss of myelin compaction was more obvious in tacrolimus-treated group (Fig. 5B).

\section{Discussion}

In the present study, rats in the three treatment groups (FTY720, tacrolimus and FTY720 + tacrolimus) were found to exhibit statistically significant improvements following $\mathrm{SCI}$, as quantified by the BBB score, the inclined plane test, footprint analysis, electrophysiological tests and electron microscopic analysis. Notably, treatment with a combination of FTY720 and tacrolimus afforded greater effect than treatment with either FTY720 or tacrolimus alone.

It was reported recently that SCI could trigger an autoimmune response to myelin basic protein (MBP) (Jones et al. 2002). MBP immunizations exacerbated neurological deficits, demyelination, and / or neuronal survival / function in animals subjected to SCI. These deficits were associated with increased numbers of infiltrating $\mathrm{T}$ cells and enhanced macrophage / microglial activation in areas of secondary injury (Jones et al. 2004). The fact that lymphocytes also responded to MBP may contribute to post-traumatic secondary degeneration (Jones et al. 2002). The effect of immunosuppression as a therapy for SCI could be explained by these observations.

Tacrolimus (also called FK506), a kind of macrolides immune inhibitor, is widely used in organ transplantation (Fukudo et al. 2009; Simmonds et al. 2009). It can promote physiological functional recovery of transplanted allogeneic limbs (Dubernard et al. 2003), and improve the locomotor function of limbs after SCI (Madsen et al. 1998; Voda et al. 2005; Pan et al. 2006a) In the present study, it was also found that tacrolimus effected statistically significant improvements after SCI, as quantified by the BBB score, the inclined plane test, footprint analysis, electrophysiological tests and electron microscopic analysis. Like cyclosporin A, tacrolimus elicits immunosuppression by inhibiting the protein phosphatase 2B (calcineurin) and subsequently blocking activation of the nuclear factor of the activated $\mathrm{T}$ cell (NFAT), thereby preventing interleukin-2 (IL-2) transcrip- tion (Ahn et al. 2007). This calcineurin inhibition is mediated by neuroimmunophilins, a family of proteins that bind to tacrolimus or cyclosporin A, referred to as tacrolimusbinding proteins (FKBP) or cyclophilins, respectively. In $\mathrm{T}$ cells, binding of tacrolimus to FKBP-12 mediates calcineurin inhibition and, consequently, immunosuppression (Clipstone and Crabtree 1992; Gold and Zhong 2004).

To our knowledge, this is the first report that a new kind of immune inhibitor, FTY720, could also exhibit statistically significant improvements in SCI, as quantified by BBB scores, the inclined plane test, footprint analysis, electrophysiological tests and electron microscopic analysis. It did not show significantly better results than tacrolimus in functional recovery after SCI though. FTY720, originally derived from the fungal natural product ISP-1 by medicinal chemistry efforts (Sasaki et al. 1994), elicits its unique immunosuppression by inducing a marked decrease in the number of peripheral blood lymphocytes, especially $\mathrm{T}$ cells (Hoshino et al. 1996). It selectively induces cell death in mature T-lymphocyte, especially CD4-positive cells, in peripheral blood without depressing bone marrow (Enosawa et al. 1996), and then decreases the number of lymphocytes (Chiba et al. 1998).

Several studies have shown that FTY720 given in combination with tacrolimus caused a synergistic effect in rat allograft models, such as skin, cardiac, liver and kidney transplantation (Xu et al. 1998; Tamura et al. 2000; Gallo et al. 2006; Lopes et al. 2008). In this study it was found that treatment with combination of FTY720 and tacrolimus had a significantly stronger effect on promoting functional recovery of spinal cord damage than treatment with either FTY720 or tacrolimus alone. As indicated in the result section, the FTY720 + tacrolimus-treated group has significantly higher BBB scores, mean angle scores and amplitude of SSEPs; greater in stride length; shorter in stride width and latency of SSEPs; less pathological changes in axons and myelin, than those of in FTY720- and tacrolimusalone-treated groups, respectively.

Tacrolimus can inhibit IL-2 transcription of activated T cells (Tocci et al. 1989; Ahn et al. 2007), and FTY720 can induce a marked decrease in the number of peripheral blood lymphocytes (especially T cells) (Yanagawa et al. 1998; Gold 2000). A marked suppression of lymphocyte infiltration and decreased levels of mRNAs for IL-2, IFN-gamma, and granzyme B can be seen with combination therapy accounting for powerful mutual effect of FTY720 and tacrolimus (Tamura et al. 2000). The greater effects on the functional recovery on SCI by the combination of FTY720 and tacrolimus than those of FTY720 or tacrolimus alone are explained by their different effects on lymphocytes and powerful mutual effect, which were involved in the development of SCI (Jones et al. 2002, 2004).

In summary, the present study has shown that FTY720 is as effective as tacrolimus in promoting the functional recovery after SCI, and that the combination of FTY720 and tacrolimus provides even better effect. The results sug- 
gest that the combination of FTY720 and tacrolimus may have potential therapeutic efficacy for treating acute SCI. Further investigations are needed to describe the detailed mechanism of the combination of FTY720 and tacrolimus on functional recovery from SCI.

\section{Acknowledgments}

This work was supported by the National Natural Science Foundation of China (Grant \#30070767 and \#30973058), the Young Talent Foundation of Jiangsu Province, China (Grants \# BK2005436 and \#RC2007060) and the Sixth Peak Talent Foundation of Jiangsu Province, China.

\section{References}

Ahn, H.S., Kim, S.E., Choi, B.H., Choi, J.S., Kim, M.J., Rhie, D.J., Yoon, S.H., Jo, Y.H., Kim, M.S., Sung, K.W., Kwon, O.J. \& Hahn, S.J. (2007) alcineurin-independent inhibition of KV1.3 by FK-506 (tacrolimus): a novel pharmacological property. Am. J. Physiol. Cell Physiol., 292, C1714-1722.

Balatoni, B., Storch, M.K., Swoboda, E.M., Schonborn, V., Koziel, A., Lambrou, G.N., Hiestand, P.C., Weissert, R. \& Foster, C.A. (2007) FTY720 sustains and restores neuronal function in the DA rat model of MOG-induced experimental autoimmune encephalomyelitis. Brain Res. Bull., 74, 307-316.

Basso, D.M., Beattie, M.S. \& Bresnahan, J.C. (1995) A sensitive and reliable locomotor rating scale for open field testing in rats. J. Neurotrauma, 12, 1-21.

Cao, Y., Lu, X.F., Han, C.L. \& Zhang, X.Y. (2005) Survival time of rats after limb allotransplantation and the combined immunosuppression of FK506 and FTY720. Chinese Journal of Clinical Rehabilitation, 22, 103-105.

Chiba, K., Yanagawa, Y., Masubuchi, Y., Kataoka, H., Kawaguchi, T., Ohtsuki, M. \& Hoshino, Y. (1998) FTY720, a novel immunosuppressant, induces sequestration of circulating mature lymphocytes by acceleration of lymphocyte homing in rats. I. FTY720 selectively decreases the number of circulating nature lymphocytes by acceleration of lymphocyte homing. J. Immunol., 160, 5037-5044.

Clipstone, N.A. \& Crabtree, G.R. (1992) Identification of calcineurin as a key signalling enzyme in T-lymphocyte activation. Nature, 357, 695-697.

Daniel, C., Sartory, N., Zahn, N., Geisslinger, G., Radeke, H.H. \& Stein, J.M. (2007) FTY720 ameliorates Th1-mediated colitis in mice by directly affecting the functional activity of $\mathrm{CD} 4^{+} \mathrm{CD} 25^{+}$regulatory T cells. J. Immunol., 178, 2458-2468.

Dorsher, P.T. \& McIntosh, P.M. (2009) Acupuncture's Effects in Treating the Sequelae of Acute and Chronic Spinal Cord Injuries: A Review of Allopathic and Traditional Chinese Medicine Literature. Evid. Based Complement. Alternat. Med., [Epub ahead of print].

Dubernard, J.M., Petruzzo, P., Lanzetta, M., Parmentier, H., Martin, X., Dawahra, M., Hakim, N.S. \& Owen, E. (2003) Functional results of the first human double-hand transplantation. Ann. Surg., 238, 128-136.

Dumont, R.J., Okonkwo, D.O., Verma, S., Hurlbert, R.J., Boulos, P.T., Ellegala, D.B. \& Dumont, A.S. (2001) Acute spinal cord injury, part I: pathophysiologic mechanisms. Clin. Neuropharmacol., 24, 254-264.

Enosawa, S., Suzuki, S., Kakefuda, T., Li, X.K. \& Amemiya, H. (1996) Induction of selective cell death targeting on mature T-lymphocytes in rats by a novel immunosuppressant, FTY720. Immunopharmacology, 34, 171-179.

Foster, C.A., Howard, L.M., Schweitzer, A., Persohn, E., Hiestand, P.C., Balatoni, B., Reuschel, R., Beerli, C., Schwartz, M. \& Billichet, A. (2007) Brain penetration of the oral immunomodulatory drug FTY720 and its phosphorylation in the cen- tral nervous system during experimental autoimmune encephalomyelitis: consequences for mode of action in multiple sclerosis. J. Pharmacol. Exp. Ther., 323, 469-475.

Fu, Q., Hue, J. \& Li, S. (2007) Nonsteroidal anti-inflammatory drugs promote axon regeneration via RhoA inhibition. J. Neurosci., 27, 4154-4164.

Fujita, T., Inoue, K., Yamamoto, S., Ikumoto, T., Sasaki, S., Toyama, R., Chiba, K., Hoshino, Y. \& Okumoto, T. (1994) Fungal metabolites. Part 11. A potent immunosuppressive activity found in Isaria sinclairii metabolite. J. Antibiot. (Tokyo), 47, 208-215.

Fukudo, M., Yano, I., Shinsako, K., Katsura, T., Takada, Y., Uemoto, S. \& Inui, K. (2009) Prospective evaluation of the bayesian method for individualizing tacrolimus dose early after living-donor liver transplantation. J. Clin. Pharmacol., 49, 789-797.

Gallo, A.P., Silva, L.B., Franco, M., Burdmann, E.A. \& Bueno, V. (2006) Tacrolimus in combination with FTY720-an analysis of renal and blood parameters. Int. Immunopharmacol., 6, 1919-1924.

Gillon, R.S., Cui, Q., Dunlop, S.A. \& Harvey, A.R. (2003) Effects of immunosuppression on regrowth of adult rat retinal ganglion cell axons into peripheral nerve allografts. J. Neurosci. Res., 74, 524-532.

Gold, B.G. \& Zhong, Y.P. (2004) FK506 requires stimulation of the extracellular signal-regulated kinase $1 / 2$ and the steroid receptor chaperone protein $\mathrm{p} 23$ for neurite elongation. Neurosignals, 13, 122-129.

Gold, B.G. (2000) Neuroimmunophilin ligands: evaluation of their therapeutic potential for the treatment of neurological disorders. Expert Opin Investing. Drugs, 9, 2331-2342.

Gruessner, R.W. (1997) Tacrolimus in pancreas transplantation: a multicenter analysis. Tacrolimus Pancreas Transplant Study Group. Clin. Transplant., 11, 299-312.

Gruner, J.A. (1992) A monitored contusion model of spinal cord injury in the rat. J. Neurotrauma, 9, 123-128.

Hauben, E., Gothilf, A., Cohen, A., Butovsky, O., Nevo, U., Smirnov, I., Yoles, E., Akselrod, S. \& Schwartz, M. (2003) Vaccination with dendritic cells pulsed with peptides of myelin basic protein promotes functional recovery from spinal cord injury. J. Neurosci., 23, 8808-8819.

Hauben, E., Ibarra, A., Mizrahi, T., Barouch, R., Agranov, E. \& Schwartz, M. (2001) Vaccination with a Nogo-A-derived peptide after incomplete spinal cord injury promotes recovery via a T-cell-mediated neuroprotective response: comparison with other myelin antigens. Proc. Natl. Acad. Sci. U.S.A., 98, 15173-15178.

Hoshino, Y., Suzuki, C., Ohtsuki, M., Masubuchi, Y., Amano, Y. \& Chiba, K. (1996) FTY720, a novel immunosuppressant possessing unique mechanisms. II. Long-term graft survival induction in rat heterotopic cardiac allografts and synergistic effect in combination with cyclosporine. Transplant. Proc., 28, 1060-1061.

Hoshino, Y., Yanagawa, Y., Ohtsuki, M., Nakayama, S., Hashimoto, T. \& Chiba, K. (1999) FTY720, a novel immunosuppressant, shows a synergistic effect in combination with FK506 in rat allograft models. Transplant. Proc., 31, 1224-1226.

Jones, T.B., Ankeny, D.P., Guan, Z., McGaughy, V., Fisher, L.C., Basso, D.M. \& Popovich, P.G. (2004) Passive or active immunization with myelin basic protein impairs neurological function and exacerbates neuropathology after spinal cord injury in rats. J. Neurosci., 24, 3752-3761.

Jones, T.B., Basso, D.M., Sodhi, A., Pan, J.Z., Hart, R.P., MacCallum, R.C., Lee, S., Whitacre, C.C. \& Popovich, P.G. (2002) Pathological CNS autoimmune disease triggered by traumatic spinal cord injury: implications for autoimmune vaccine therapy. J. Neurosci., 22, 2690-2700.

Jou, I.M., Lai, K.A., Shen, C.L. \& Yamano, Y. (2000) Changes in conduction, blood flow, histology, and neurological status fol- 
lowing acute nerve-stretch injury induced by femoral lengthening. J. Orthop. Res., 18, 149-155.

Kaymaz, M., Emmez, H., Bukan, N., Dursun, A., Kurt, G., Pasaoglu, H. \& Pasaoglu, A. (2005) Effectiveness of FK506 on lipid peroxidation in the spinal cord following experimental traumatic injury. Spinal Cord, 43, 22-26.

Li, S., Liu, B.P., Budel, S., Li, M., Ji, B., Walus, L., Li, W., Jirik, A., Rabacchi, S., Choi, E., Worley, D., Sah, D.W., Pepinsky, B., Lee, D., Relton, J. \& Strittmatter, S.M. (2004) Blockade of Nogo-66, myelin-associated glycoprotein, and oligodendrocyte myelin glycoprotein by soluble Nogo-66 receptor promotes axonal sprouting and recovery after spinal injury. J. Neurosci., 24, 10511-10520.

Lo, C.G., Xu, Y., Proia, R.L. \& Cyster, J.G. (2005) Cyclical modulation of sphingosine-1-phosphate receptor 1 surface expression during lymphocyte recirculation and relationship to lymphoid organ transit. J. Exp. Med., 201, 291-301.

Lopes, C.T., Gallo, A.P., Palma, P.V., Cury, P.M. \& Bueno, V. (2008) Skin allograft survival and analysis of renal parameters after FTY720 + tacrolimus treatment in mice. Transplant. Proc., 40, 856-860.

Lopez-Vales, R., Garcia-Alias, G., Fores, J., Udina, E., Gold, B.G., Navarro, X. \& Verdu, E. (2005) FK506 reduces tissue damage and prevents functional deficit after spinal cord injury in the rat. J. Neurosci. Res., 81, 827-836.

Madsen, J.R., MacDonald, P., Irwin, N., Goldberg, D.E., Yao, G.L., Meiri, K.F., Rimm, I.J., Stieg, P.E. \& Benowitz, L.I. (1998) Tacrolimus (FK506) increases neuronal expression of GAP-43 and improves functional recovery after spinal cord injury in rats. Exp. Neurol., 154, 673-683.

Metz, G.A., Merkler, D., Dietz, V., Schwab, M.E. \& Fouad, K. (2000) Efficient testing of motor function in spinal cord injured rats. Brain Res., 883, 165-177.

Pan, F., Chen, A.M., Guo, F.J. \& Zhu, C.L. (2006a) Effect of tacrolimus on apoptosis and expression of heat shock protein 70 after acute spinal cord injury in rats. Zhonghua Wai Ke Za Zhi, 44, 1708-1712.

Pan, F., Chen, A.M., Guo, F.J. \& Zhu, C.L. (2006b) Experimental study of effect of low-dose tacrolimus on acute spinal cord injury in rats. Chinese journal of spine and spinal cord, 12, 927-931. (in Chinese)

Rivlin, A.S. \& Tator, C.H. (1977) Objective clinical assessment of motor function after experimental spinal cord injury in the rat. J. Neurosurg., 47, 577-581.

Rossignol, S., Schwab, M., Schwartz, M. \& Fehlings, M.G. (2007)
Spinal Cord Injury: time to move? J. Neurosci., 27, 1178211792.

Saadoun, S., Bell, B.A., Verkman, A.S. \& Papadopoulos, M.C. (2008) Greatly improved neurological outcome after spinal cord compression injury in AQP4-deficient mice. Brain, 131, 1087-1098.

Sasaki, S., Hashimoto, R., Kiuchi, M., Inoue, K., Ikumoto, T. Hirose, R., Chiba, K., Hoshino, Y., Okumoto, T. \& Fujita, T. (1994) Fungal metabolites. Part 14. Novel potent immunosuppressants, mycestericins, produced by Myceria sterilia. $J$. Antibiot. (Tokyo), 47, 420-433.

Shiraishi, S., Yanagita, T., Kobayashi, H., Uezono, Y., Yokoo, H., Minami, S.I., Takasaki, M. \& Wada, A. (2001) Up-regulation of cell surface sodium channels by cyclosporin A, FK506, and rapamycin in adrenal chromaffin cells. J. Pharmacol. Exp. Ther., 297, 657-665.

Simmonds, J., Dewar, C., Dawkins, H., Burch, M. \& Fenton, M. (2009) Tacrolimus in pediatric heart transplantation: ameliorated side effects in the steroid-free, statin era. Clin. Transplant., 23, 415-419.

Tamura, A., Li, X.K., Funeshima, N., Enosawa, S., Amemiya, H., Kitajima, M. \& Suzuki, S. (2000) Immunosuppressive therapy using FTY720 combined with tacrolimus in rat liver transplantation. Surgery, 127, 47-54.

Tocci, M.J., Matkovich, D.A., Collier, K.A., Kwok, P., Dumont, F., Lin, S., Degudicibus, S., Siekierka, J.J., Chin, J. \& Hutchinson, N.I. (1989) The immunosuppressant FK506 selectively inhibits expression of early $\mathrm{T}$ cell activation genes. J. Immunol., 143, 718-726.

Voda, J., Yamaji, T. \& Gold, B.G. (2005) Neuroimmunophilin ligands improve functional recovery and increase axonal growth after spinal cord hemisection in rats. J. Neurotrauma, 22, 1150-1161

Xu, M., Pirenne, J., Antoniou, S., Gunson, B., D’Silva, M. \& McMaster, P. (1998) FTY720 compares with FK 506 as rescue therapy in rat heterotopic cardiac transplantation. Transplant. Proc., 30, 2221-2222.

Yanagawa, Y., Sugahara, K., Kataoka, H., Kawaguchi, T., Masubuchi, Y. \& Chiba, K. (1998) FTY720, a Novel immunosuppressant, induces sequestration of circulating mature lymphocytes by acceleration of lymphocyte homing in rats. II. FTY720 prolongs skin allograft survival by decreasing T cell infiltration into grafts but not cytokine production in vivo. J. Immunol., 160, 5493-5499. 